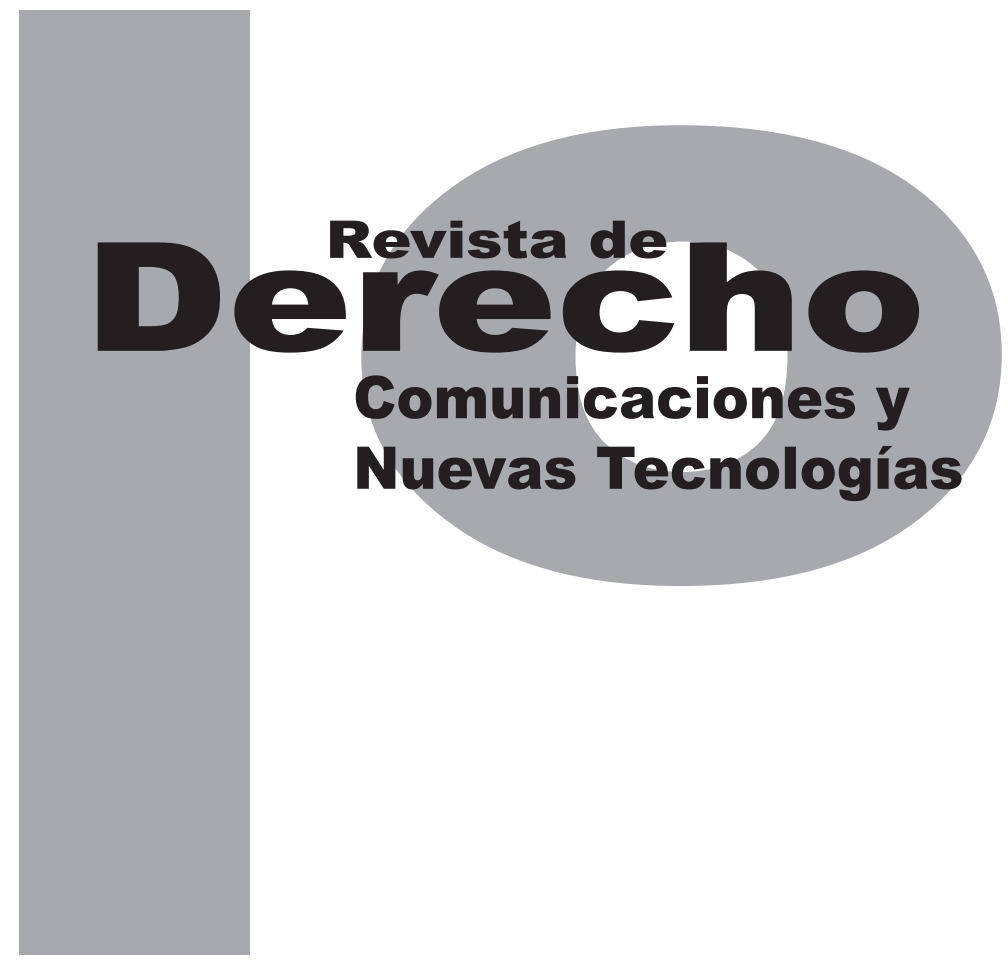

\title{
EL DERECHO, LA SOCIEDAD DE LA INFORMACIÓN Y EL PRINCIPIO DE LA NEUTRALIDAD DE RED: CONSIDERACIONES SOBRE EL MERCADO Y EL ACCESO A LA INFORMACIÓN
}

\author{
THIAGO LOPES MATSUSHITA \\ Artículo de reflexión \\ Universidad de los Andes \\ Facultad de Derecho
}

Revista de Derecho, Comunicaciones y Nuevas Tecnologías

No.11, Enero - Junio de 2014. ISSN 1909-7786 


\title{
El derecho, la sociedad de la información y el principio de la neutralidad de red: consideraciones sobre el mercado y el acceso a la información
}

\section{Resumen}

La sociedad de la información en la cual vivimos se caracteriza por el avance sin precedentes de las tecnologías de la información y comunicación, cuyo aparato instrumental promueve nuevas formas de relacionamiento humano, consustanciadas con el proceso continuo y dinámico de la globalización. En este sentido, el libre acceso a la información se erige al nivel de derecho humano fundamental, contribuyendo a que la economía y la política confluyan como poderes cada vez más democratizados, causando la generación de nuevas configuraciones del Estado y remodelando el mercado, dominado predominantemente por algunos conglomerados económicos. Así, urge la necesidad de protección de estos derechos, con la garantía del derecho a la dignidad de la persona humana, presupuesto basilar de toda sociedad cuyo orden se diga democrático y de derecho.

Palabras clave: Sociedad de la información, neutralidad de red, jushumanismo normativo.

\section{The law, the society of information and the net neutrality principle: Considerations about the market and access to information}

\begin{abstract}
This article aims to promote an approach to the Society of Information and the technological advancements that support it under the perspective of Law and the principle of net neutrality. To achieve that, we avail of observing the globalized economy through the inherent dimension provided by the internet and all the other information technologies and in which their bias allows the democratic development of society through the incorporation of assumptions of the normative jus-humanism theory, objectifying the utmost realization of the objective rights of human dignity.
\end{abstract}

Keywords: Society of Information. Net neutrality. Normative Jus-humanismo

\section{O direito, a sociedade da informação e o princípio da neutralidade de rede: considerações sobre o mercado e o acesso à informação}

\section{Resumo}

A Sociedade da Informação na qual vivemos se caracteriza pelo avanço sem precedentes das tecnologias de informação e comunicação, cujo aparato instrumental promove novas formas de relacionamento humano, consubstanciadas pelo processo contínuo e dinâmico da globalização. Neste viés, o livre acesso à informação é erigido ao patamar de direito humano fundamental, contribuindo para que a economia e a política confluam-se como poderes cada vez mais democratizados, provocando a geração de novas configurações do Estado e remodelando o mercado dominado predominantemente por alguns conglomerados econômicos. Assim, urge, na contemporaneidade, a necessidade de proteção de tal direito com o viés de garantia do direito à dignidade da pessoa humana, pressuposto basilar de toda sociedade cuja ordem se diga Democrática e de Direito.

Palavras-chave: Sociedade da Informação. Neutralidade de rede. Jus-humanismo normativo. 


\section{SUMARIO}

Introducción - I. DIFUSIÓN DEL PODER Y LIBRE ACCESO A LA INFORMACIÓN - II. EL MERCADO Y LA SOCIEDAD DE LA INFORMACIÓN - III. PRINCIPIO DE LA NEUTRALIDAD DE RED - IV. LA ECONOMÍA Y LA BASE INMATERIAL DE LA SOCIEDAD - V. CONCLUSIONES - Referencias - Cibergrafía. 


\title{
El derecho, la sociedad de la información y el principio de la neutralidad de red: consideraciones sobre el mercado y el acceso a la información ${ }^{1}$
}

\author{
Thiago Lopes Matsushita²
}

\section{Introducción}

El presente artículo aborda la sociedad de la información y de los avances tecnológicos que le competen bajo la óptica del derecho y del principio de la neutralidad de red (net neutrality). Para conseguirlo, analizaremos la economía a través del enfoque ofrecido por las tecnologías de la información y comunicación, observando el derecho a la información y el desarrollo de la sociedad globalizada bajo el sesgo de la teoría del jushumanismo normativo.

En este sentido, consideramos la "globalización" como un concepto que, en términos de David Held y Anthony McGrew (2001),

Cómo citar este artículo: Lopes Matsushita, T. (Junio, 2014). El derecho, la sociedad de la información y el principio de la neutralidad de red: consideraciones sobre el mercado y el acceso a la información. Revista de Derecho, Comunicaciones y Nuevas Tecnologías, 11.

2 Maestro y doctor en Derecho Económico de la Pontificia Universidad Católica de São Paulo, PUC/SP. Profesor de pregrado, maestría y doctorado de la Facultad de Derecho de la PUC/SP. Vicecoordinador del curso de pregrado en Derecho de la Facultad de Derecho de la PUC/SP.
[...] denota mucho más que la ampliación de relaciones y actividades sociales atravesando regiones y fronteras. Sugiere una magnitud o intensidad creciente de flujos globales, de tal manera que Estados y sociedades quedan cada vez más enredados en sistemas mundiales y redes de interacción. En consecuencia, acontecimientos y fenómenos distantes pueden pasar a tener serios impactos internos, mientras que acontecimientos locales pueden generar repercusiones globales de peso. (p. 12). Traducción propia

En este sentido, la globalización es un trayecto de acortamiento de distancias entre los seres humanos a nivel económico, cultural, político y social, a través de un proceso que, principalmente, en los últimos treinta años se dio a una velocidad jamás vista, como consecuencia básicamente del desarrollo de la tecnología de la información y comunicación, especialmente de Internet, capaz de proporcionar virtualmente el encuentro de una persona (o grupo de personas) con cualquier otra del globo, en cualquier momento, lo que ocasionó la ruptura de las bar- 
reras físicas. Tal rompimiento se da principalmente con la evolución de la velocidad de desplazamiento del propio pensamiento, tornándose un instrumento técnico. A propósito, Marilena Chauí propone un análisis de la sociedad actual de la información, diferenciando su proceso inherente de desarrollo tecnológico de aquellos ocurridos en el pasado. En sus términos:

La gran mudanza tecnológica traída por la noción de información es que el paradigma de objeto técnico, o de objeto tecnológico, es el sistema nervioso y el cerebro humano. Por tanto, lo que se amplió, lo que se tornó el instrumento por excelencia fue el pensamiento. Es la primera vez en la historia de la humanidad que el pensamiento se torna, él propio, un instrumento técnico. Y es por eso que los objetos tecnológicos que nosotros conocemos son autómatas, porque lo autómata es exactamente aquello que es característico de nuestro pensamiento, o sea, la capacidad de iniciar su actividad por cuenta propia, de autocorregirse, de reverse y de proseguir. (2013). Traducción propia

Junto con este desarrollo sin precedentes en la historia, el conocimiento se tornó mundializado, alterando sobremanera la forma en que los individuos y las comunidades en que son partícipes enfrentan la comunicación, inclusive en la esfera jurídica y de mercado.

Respecto al mercado, la teoría de la obsolescencia planeada, ${ }^{3}$ en cuanto paradigma del ca-

3 Sobre la obsolescencia planeada, valen las palabras de Valquíria Padilha y Renata Cristina A. Bonifácio: "Planear cuando un producto va a fallar o tornarse viejo, programando su fin inclusive antes de la acción de la naturaleza y del tiempo de uso es la obsolescencia planeada. Se trata de la estrategia de establecer una fecha de muerte de un producto, sea por medio del mal funcionamiento o envejecimiento frente pitalismo desde el inicio del siglo XX, alcanzó al saber al volverlo "fuerza productiva" (2013). No se diferencia en cuanto entidad autónoma, pero, siguiendo a Chauí, se incorpora directamente en la economía de la denominada actualmente "sociedad del conocimiento". El capitalismo, de esta forma, según Sérgio Amadeu da Silveira, no vive más de la lógica de la reproducción, sino de la invención permanente, donde aquella obsolescencia acaba siendo siempre provocada.

A su vez, en el ámbito jurídico, el libre acceso a la información para algunos autores, como Paulo Bonavides, se clasifica como un derecho fundamental. La propia Declaración Universal de los Derechos Humanos de 1948 proclama, en su artículo 19, que "Toda pessoa tem direito à liberdade de opinião e expressão; este direito inclui a liberdade de, sem interferência, ter opiniões e de procurar, receber e transmitir informações e idéias por quaisquer meios e independentemente de fronteiras".

En la misma línea sigue el Pacto Internacional de los Derechos Civiles y Políticos que, en su artículo 19, prescribe:

\footnotetext{
a las tecnologías más recientes. Esa estrategia fue discutida como solución para la crisis de 1929. El concepto inició alrededor de 1920, cuando fabricantes comenzaron a reducir a propósito la vida de sus productos para aumentar la venta y el lucro. La primera víctima fue la lámpara eléctrica, con la creación del primer cartel mundial (Phoebus) para controlar la producción. Sus miembros percibieron que las lámparas que duraban mucho no eran ventajosas. La primera lámpara inventada duraba 1500 horas. En 1924, las lámparas duraban 2500 horas. En 1940, el cartel consiguió su objetivo: el patrón de vida de las lámparas era de 1000 horas. Para que ese objetivo se consiguiera, fue necesario fabricar una lámpara más frágil". Padilha, V., Bonifácio, R. C. A. Produção da destruição. Obsolescência planejada: armadilha silenciosa na sociedade de consumo. Le monde diplomatique Brasil. 2 de septiembre de 2013. Disponible en https://www.diplomatique.org. br/artigo.php?id=1489.
} 
Toda persona tiene derecho a la libertad de expresión; este derecho comprende la libertad de buscar, recibir y difundir informaciones e ideas de toda índole, sin consideración de fronteras, ya sea oralmente, por escrito o en forma impresa o artística, o por cualquier otro procedimiento de su elección.

En este sentido, a fin de garantizar la libertad de expresión y un orden jurídico democrático, se tramita en el Congreso Nacional Brasilero el proyecto de ley de la Cámara núm. 21 denominado Marco Civil de Internet que, inclusive, dispone en su artículo 7 que el acceso a Internet es esencial para el ejercicio de la ciudadanía.

\section{DIFUSIÓN DEL PODER Y LIBRE ACCESO A LA INFORMACIÓN}

El libre acceso a la información puede servir de instrumento para la emancipación autónoma del individuo y de la colectividad, contribuyendo inclusive al desarrollo de la propia noción de democracia mediante la inserción social a través de la apertura a la posibilidad, cada vez mayor, de transparencia del poder público y de revolución del proceso educativo. La relación de poder inclusive en el ambiente democrático ya no es capaz de mantenerse como antes, o sea, estructurada sobre la base de la restricción de la información, al tornarse cada vez más transparente la publicidad de actos que, de inicio, deberían siempre ser públicos, y hasta de actividades cuya característica eminentemente privada desaparece en la sociedad contemporánea de la información.
El poder también se torna más difuso debido, inclusive, a la posibilidad de que cualquiera genere conocimiento, productos y servicios para el mercado. La teoría de Chris Anderson, sobre la cual nos apoyamos, es que vivimos en un mundo en que, en sus palabras, "any kid with an idea and a laptop can create the seeds of a world-changing company, just look at Mark Zuckerberg and Facebook or any one of thousands of other web startups hoping to follow his path" (Anderson, 2012, p. 7). Esto conduce a la idea de que la belleza de la web es que democratiza los dos instrumentos de invención y de producción (Anderson, 2012, p. 7). El autor resalta inclusive que antes de Internet, "it used to be hard to change the world with an idea alone. You can invent a better mousetrap, but if you can't make it in the millions, the world won't beat a path to your door. As Marx observed, power belongs to those who control the means of production" (Anderson, 2012, p. 5).

Los servicios generados por las unidades elementales del mundo digital, los bits, según Anderson, son más baratos y viajan por el mundo a una velocidad mayor, jamás vista, construyendo redes de servicios capaces de ofrecer sus productos en territorios considerados antiguamente distantes. Reformulando al mundo, la forma de comunicarse y de relacionarse inclusive con el propio capitalismo, "bits have changed the world" (Anderson, 2012, p. 8). En este sentido, para Anderson, estamos en la era de la nueva revolución industrial. Por lo tanto, hay una relación entre la Internet y los proyectos de producción de bienes de consumo: 
These projects represent the ideas, dreams, and passions of millions of people. Most never leave the home, and that's probably no bad thing. But one of the most profound shifts of the web age is that there is a new default of sharing online. If you do something, video it. If you video something, post it. If you post something, promote it to your friends. Projects, shared online, become inspiration for others and opportunities for collaboration. Individual Makers, globally connected this way, become a movement. Millions of Diyers [Do It Yourselfers], once working alone, suddenly start working together. (p. 13)

Además, la democracia es conducida en el sentido de traer el poder efectivamente a la sociedad: el poder de decisión y de elección política e, inclusive, económica, aun frente a las grandes empresas que controlan el mercado en el ámbito mundial. O sea, si se pensaba que la política, especialmente en el siglo XX, se había desplazado hacia la fuerza de los conglomerados económicos globalizados, actualmente y de aquí en adelante estos están destinados a perder su lugar dominante en las decisiones políticas, para una dispersión de poder a la sociedad tecnológica en que vivimos.

Tal dispersión puede inclusive alterar el concepto contemporáneo de Estado por el del surgimiento paralelo de formas de colaboración social, eminentemente propiciadoras de nuevas y creativas reglas de mercado y de relación interpersonal. Es urgente la adopción de una estructura de Estado más flexible que reciba otras formas de regulación y de juridicidad, principalmente si no se quiere cometer el constante error de extinguirse en la "ambivalencia" permanente y cada vez más evidente, utilizando la terminología de Zygmunt Bauman.

Así se entiende la ambivalencia: "Posibilidad de atribuir a un objeto o evento más de una categoría" (Bauman, 1999, p. 9). La búsqueda permanente de la modernidad de exterminar la ambivalencia trae la necesidad constante de separar, catalogar, dividir, generando la intolerancia, tornándose en "la inclinación natural de la práctica moderna” (Bauman, 1999, p. 16). En palabras de Bauman:

Lo otro del Estado moderno es la tierra de nadie o sin respuesta: la sub o sobredefinición, el demonio de la ambigüedad. Una vez que la soberanía del Estado moderno es el poder de definir y de hacer que las definiciones se acojan, todo lo que se autodefine o que escapa a la definición asistida por el poder es subversivo. Lo otro de esa soberanía son áreas prohibidas, de agitación y desobediencia, de colapso de la ley y del orden.

\section{$[\ldots]$}

En el reino político, expurgar la ambivalencia significa segregar o deportar a los extraños, sancionar algunos poderes locales y colocar fuera de la ley a aquellos no sancionados, llenando así las "brechas de la ley". (pp. 16-33). Traducción propia

De esta forma, en la contemporaneidad se le debe prestar atención a la posibilidad de nuevas configuraciones del Estado moderno e incluir nuevas formas de sociabilidad que surjan con la sociedad de la información, bajo el riesgo 
de que la corriente de segregación alcance a aquellos que se fortalecen a través y principalmente por los medios hodiernos de producción de conocimiento e innovación, pudiendo llevar a una consecuente deslegitimación del propio poder estatal. Esas nuevas formas de sociabilidad se diseñan principalmente con el desarrollo de las tecnologías de la información y comunicación. Así, con la intensa globalización de dichas tecnologías, el Estado debe adecuarse a la producción de nuevas formas de revalidación constante de su papel en la actualidad, a fin de mantenerse como instrumento edificador de la democracia y de los derechos fundamentales. Sobre las nuevas configuraciones sociales, vaIen las palabras de Pierre Lévy (2001):

La tendencia se diseña claramente. En los primeros decenios del siglo XXI, más del $80 \%$ de los seres humanos tendrán acceso al ciberespacio y se servirán de él cotidianamente. La mayor parte de la vida social tomará prestado ese medio. Los procesos de concepción, producción y comercialización serán integralmente condicionados por su inmersión en el espacio virtual. Las actividades de investigación, de aprendizaje y de ocio serán virtuales o comandadas por la economía virtual. El ciberespacio será el epicentro del mercado, el lugar de creación y de adquisición de conocimientos, el principal medio de comunicación y de la vida social. (p. 51). Traducción propia

La importancia del desarrollo de tales tecnologías, por tanto, es fundamento de la sociedad contemporánea, pues altera drásticamente las relaciones interpersonales en todas sus dimensiones. No es por nada que Paulo Bonavides (2004), por ejemplo, ya se refirió al derecho a la información como un derecho fundamental de cuarta generación. En sus términos:

Son derechos de cuarta generación el derecho a la democracia, el derecho a la información y el derecho al pluralismo. De ellos depende la concretización de la sociedad abierta del futuro, en su dimensión de máxima universalidad, para la cual parece el mundo inclinarse en el plano de todas las relaciones de convivencia. Traducción propia

Elevar el derecho a la información a la categoría de derecho humano equivale a decir que el ciudadano tiene el derecho a adquirir información por los instrumentos adecuados y que el Estado tiene el deber de transparencia a través de la publicidad de sus actos y de la disponibilidad de medios tecnológicos para el alcance democrático de las informaciones prestadas. Además, los derechos humanos sobrepasan la línea vertical de la relación ciudadano/Estado. Conforme ya dijimos en otro momento:

En ese sentido la aplicación de los derechos humanos debe ocurrir de forma vertical, cuando el Estado es quien compromete los medios necesarios para la promoción de la inclusión del ser humano en condiciones en las que salga a la búsqueda de sus deseos, buscando la satisfacción de sus necesidades y, también, de forma horizontal, cuando no solo el Estado es quien debe promover esos medios necesarios, mas todos los individuos de la sociedad deben contribuir para la inclusión de todos [...]. (Matsushita, 2012, p. 93). Traducción propia

Vale resaltar que las dimensiones (o generaciones) de derechos humanos no son estáticas, 
pero sí una construcción cultural e histórica que implica avances, adiciones de nuevos derechos, conforme al desarrollo y la coyuntura de la épo$\mathrm{ca}$, siempre en forma de densidad, o sea, todas las dimensiones deben ser observadas conjuntamente, sin exclusión de ninguna, en pro de la dignidad de la persona humana.

\section{EL MERCADO Y LA SOCIEDAD DE LA INFORMACIÓN}

Entendemos, siguiendo a la mayoría de la posición doctrinaria, que el proceso de globalización está cada vez más encaminado a la concentración de mercado en manos de pocos conglomerados económicos. Esa es una tendencia que, conforme Lévy, solo tiende a aumentar. Sin embargo, vale considerar que el mercado, frente a las patentes y los derechos autorales, por ejemplo, está creciendo y tiende a la gratuidad de diversos productos, en el sentido de creación inclusive de mercados paralelos establecidos en las manos de pequeños negociantes en pro de la democratización de diversos productos que, de inicio, estaban sometidos a las manos de grandes conglomerados empresariales. Chris Anderson (2012) utiliza el ejemplo brasilero de la banda Calypso, cuya venta de cedés es ampliamente efectuada a través de la piratería, y del caso de pago de regalías a la producción de medicamentos para VIH-positivo, para sustentar su tesis:

Ninety percent of the bands have no record contract and no label. They don't need one. Letting others get their music for free creates a bigger industry than charging ever could. This is something that Brazil understands better than most: Its culture minister until 2008, the pop star Gilberto Gil, has released his music under a free Creative Commons license (including in a $C D$ we distributed for free with Wired). As in China, the drive toward Free in Brazil goes far beyond music. In 1996, in response to Brazil's alarming rate of AIDS infection, the government of then- president Fernando Henrique Cardoso guaranteed distribution of the new retroviral drug cocktails to all HIV carriers in the country. Five years later, with the AIDS rate dropping, it was clear that the plan was wise but -at the prices being charged for the patented drugs in the cocktail- utterly unsustainable. So Brazil's health minister went to the key patent holders, the U.S. pharmaceutical giant Merck and the Swiss firm Roche, and asked for a volume discount. When the companies said no, the minister raised the stakes. Under Brazilian law, he informed them, he had the power in cases of national emergency to license local labs to produce patented drugs, royalty-free, and he would use it if necessary. The companies caved, and prices fell by more than 50 percent. Today, Brazil has one of the largest generic drug industries in the world. Not free, but royalty-free, an approach to intellectual property rights that the industry shares with the tecnobrega DJs. (p. 169)

De esta forma, a pesar de que la concentración de poder en las grandes empresas ha aumentado, la escasez, como pilar de lucro en el control de la oferta y de la demanda, se torna cada vez más una coyuntura producida artificialmente que una realidad de mercado. La abundancia es la palabra de orden para el gran grupo de productos. Los productos escasos no fueron solamente sustituidos porque la escasez continúa 
siendo fuente de lucro. La información era un producto que estaba sometido a este orden. No obstante, no se encuadra más en la sociedad tecnológica hodierna cuyo avance camina hacia la producción de información en escala infinitesimal: "The Internet brought the end of information scarcity" (Anderson, 2012, p. 173).

Así, frente a tal proceso de democratización es difícil imaginar, teniendo en cuenta la facilidad de acceso a las informaciones, que la política tenderá a no atender las reivindicaciones populares que gradualmente llegan a las calles, según pudimos verificar en los movimientos de junio de 2013 aquí en nuestro país. Así, afirma Willis Santiago Guerra Filho (2013) sobre tales manifestaciones:

La nación que estamos viendo nacer con el movimiento de las multitudes en las calles de nuestro país, al final del primer cuarto de siglo de vigencia de la Constitución de la República de 1988, la Constitución que antepuso los derechos al Estado, es la nación de los que sabemos que no estamos teniendo tales derechos asegurados, la nación de los que saben que tienen derechos y garantías por el simple hecho de haber nacido, de existir, pero que no basta que eso sea dicho o escrito, pues es necesario que sea hecho, hecho por nosotros, y no de ellos para nosotros, pues así hacen más para ellos, y menos por nosotros. Traducción propia

Es un hecho que, además, frente a la eclosión en las calles de esos movimientos, se verificó una pluralidad de reivindicaciones que reflejaron el descontento general de la población y de la segmentación inherente a la sociedad contemporánea. En este sentido, tal segmentación, que lleva a una pluralidad de deseos y opiniones, como no podría dejar de ser, es reverberación natural de la propia estructura inherente al mercado capitalista, siendo que este tiende a una cada vez mayor diversificación de sus productos y especialización en nichos siempre más singularizados, con el fin de atender todo tipo de demanda. Como resalta Lévy (2001):

El mejor mercado es necesariamente aquel que propone más elecciones, aquel que ofrece también los mejores medios para tomar conocimiento de las elecciones y para compararlas. Fue ese apetito por la elección, esa atracción por la libertad, lo que hizo que los hombres se agruparan en sociedades cada vez más numerosas, en la medida en que sus progresos económicos lo permitían [...]. Ahora, ellos están reagrupándose dentro de una inmensa ciudad virtual, allá donde encontramos más elecciones, allá donde podemos encontrar a todo el mundo, allá donde encontramos los mejores mercados, inclusive, y sobre todo, los mercados de la información, del conocimiento, del relacionamiento y de la diversión. Consecuentemente, los trabajos virtuales comienzan a proliferar: ellos nos permitirán habitar la gran ciudad planetaria. (p. 53) (subrayado del autor, traducción propia).

El control de esa economía virtual no se encuentra más centrado en los aparatos concretos de tecnología que determinada empresa por ventura posea, pero sí en la noción de creatividad, de bienes inmateriales, en cuya lógica la escasez se encuentra ausente y que adquieren gran valor hasta el punto que empresas como Google, centrada en la noción de profusión de información y de saberes, es una de las más valorizadas 
del mundo. O sea, partimos de la palabra de Silveira, para quien "el control de esa economía es el control de la inteligencia" (2013). En este sentido, para Chauí, estamos frente a una situación inédita:

Las experiencias mundiales de las redes sociales muestran el potencial libertario, el potencial democrático, la manera de quebrar el monopolio de las grandes empresas de comunicación, la manera de hacer con que usted tenga derecho y la libertad de pasar las informaciones que usted quiera, de escribir, de mandar para las personas. Esto está contrapuesto a un esquema de vigilancia, de control y de transformación de cada uno de nosotros en una mercancía que las empresas compran. (2013). Traducción propia

Chauí dice esto respecto al proceso de negociación y distribución de datos privados entre empresas, con el propósito de, conforme el perfil del individuo, direccionarle propagandas de productos y servicios que puedan interesarle. Nuestros datos (informaciones personales), como dice Silveira, son un activo económico (2013). Eso permite desde el direccionamiento de sitios por Google hasta el surgimiento de los indeseados spams. La manipulación de los datos privados, según lo expuesto por los medios en el caso Snowden, es inclusive agenda de sectores de espionaje del Gobierno norteamericano. ${ }^{4}$ Así,

4 Conferir, e. g., Pariz, T. Brasil clasifica como 'primer paso' decisión de Obama de prohibir el espionaje de aliados. Jornal Estadão. Disponible en http://www.estadao.com.br/noticias/geral,brasil-classifica-como-primeiro-passo-decisao-de-obama-de-proibir-espionagem-de-aliados, $1120378,0$. htm ; Robertson, J. Bloomberg News. Trad. de Celso Paciornik. Datos de usuarios de aplicativos no tienen protección. Jornal Estadão. 3 de febrero de 2014. Disponible en http://blogs.estadao. com.br/link/dados-de-usuarios-de-aplicativos-nao-tem-protecao/. acompañamos los comentarios de Fábio Konder Comparato:

Es lamentable, pues, reconocer que el derecho a la privacidad tiende a transformarse, en la actual era de la informática, en piadosa ficción, en caso de que no logre crearse una institución internacional capaz de sobreponerse a esa novísima forma de imperialismo (2008, p. 313).

Sin embargo, la presidenta de Brasil, Dilma Rousseff, va a anunciar el día 23 de abril, durante un evento con representantes de decenas de países, una especie de "Marco Civil del Internet Mundial", con la intención de reducir el poder de gobiernos en la regulación y gobernanza del Internet, a fin de dar mayores garantías a la privacidad de los usuarios (Villaverde, 2014). El contenido del documento todavía no está formalmente disponible, no obstante, en las palabras de Clelio Campolina, ministro brasileño de Ciencia, Tecnología e Información,

no existe una institucionalidad mundial para regular el comportamiento de los ciudadanos en todos los países, cada uno tiene que tener el suyo, pero necesitamos construir un sentido común. Quedar de la forma que está llevará a la barbarie. Estamos iniciando un proceso irreversible. (Villaverde, 2014)

Ese proceso reversible tiende inclusive a influenciar el funcionamiento de la economía digital que se expande a una velocidad sorprendente. El comercio, como dice Lévy (2001, p. 54), está realizando "la unidad concreta de la humanidad", radicalmente afirmando que tal hecho es "lo que ni las grandes religiones, ni la instrucción pública, ni la declaración universal 
de los derechos humanos, ni el simple sentido común habían podido construir":

De ahora en adelante, y poco a poco, el comercio de las ideas se fundirá con el comercio en general, hasta no poder más distinguirse de él. En una economía global en que el conocimiento se tornó el principal factor de concepción, de producción, de venta y, finalmente, el principal producto, cualquier comercio es un comercio de ideas más o menos objetivadas.

El planeta solidario está construyéndose por la web y su economía virtual. El crecimiento de la web "es" el proceso de toma de conciencia $-\mathrm{y}$ de realización- de su unidad por la humanidad [...]. (Lévy, 2001, p. 55). Traducción propia

No obstante, ese proceso tan positivamente previsto por Lévy puede estar comprometido, por lo menos, en el aspecto de la democracia del conocimiento que Internet tiene la posibilidad de ofrecer a corto y mediano plazo.

\section{PRINCIPIO DE LA NEUTRALIDAD DE RED}

Decimos eso basados en el reciente juicio de la Corte Federal norteamericana en el caso Verizon Communications Inc. v. FCC (Federal Communication Commission) sobre las regulaciones de esta comisión frente al principio de la net neutrality. Tal principio se refiere a la idea de que toda información debería ser tratada igualmente. Eso significa que no hay ningún tratamiento preferencial para tipos específicos de contenido, ciertos usuarios, compañías, o modos de comunicación (Estes, 2014).
Net neutrality advocates like to use the metaphor of cars on a highway, where cars represent internet traffic and the road represents the network. Left alone (read: neutral) any vehicle can zoom down whichever lane on the highway it wants without interference so long as the lane is open. But what if someone built fast lanes where only certain types of cars were allowed to travel? And what if someone toll booths along the road that already exists, just so that other cars had to pay extra to use the road? What if some cars were kicked off the road completely? (Estes, 2014)

Lo que busca la empresa de telecomunicaciones estadounidense, vencedora en el proceso, es un llamado two-sided market, envolviendo el pago por el servicio de Internet por suscriptores y por compañías que los quieren alcanzar, conforme los comentarios de Helgi Walker, abogado de Verizon (Zajac y Shields, 2014). Silberman, juez federal de la corte americana, argumentó a favor de Verizon que "this regulation essentially provides an economic preference to a politically powerful constituency, a constituency that, as is true of typical rent seekers, wishes protection against market forces" (Zajac y Shields, 2014). $Y$ que, en esa medida, "the Commission does not have authority to grant such a favor" (Zajac y Shields, 2014).

Es decir, invariablemente acaba adentrándose en la gran cuestión que envuelve la posibilidad de intervención estatal de cara al denominado free market, en el caso de las empresas servidoras de banda ancha. La FCC ya admitió que va a apelar la decisión en pro de los consumidores y de la libertad de elección hoy usufructuada por 
Internet sin restricciones, como el del contenido y los propios usuarios. En este sentido, tendemos a no estar de acuerdo con Robert Pepper (2007), exjefe de Policy Development en la FCC, quien argumenta:

The supporters of net neutrality regulation believe that more rules are necessary. In their view, without greater regulation, service providers might parcel out bandwidth or services, creating a bifurcated world in which the wealthy enjoy first-class Internet access, while everyone else is left with slow connections and degraded content.

That scenario, however, is a false paradigm. Such an all-or-nothing world doesn't exist today, nor will it exist in the future. Without additional regulation, service providers are likely to continue doing what they are doing. They will continue to offer a variety of broadband service plans at a variety of price points to suit every type of consumer.

Realmente, a nuestro entender, dividir Internet y sus usuarios mediante el contenido sí sería restringir a los menos abastecidos de acceso a una mayor cantidad y calidad de información y conocimiento, bifurcando efectivamente el mundo y reduciendo el potencial democrático de esta tecnología. A partir del momento en que excluye a ciertas personas el acceso de determinados contenidos, la información vuelve a ser un privilegio, entrando nuevamente en la lógica del capitalismo libre: libre para generar segregación y desigualdades insustentables bajo cualquier punto de vista, menos el de la exacerbación de lucro. De esta forma, compartimos los comentarios de Lévy (1999):
Cada nuevo sistema de comunicación fabrica sus excluidos. No había iletrados antes de la invención de la escritura. La impresión y la televisión introdujeron la división entre aquellos que publican o están en los medios y los otros. Como ya observé, se estima que apenas poco más de $20 \%$ de los seres humanos posee un teléfono. Ninguno de esos hechos constituye un argumento serio contra la escritura, la impresión, la televisión o el teléfono. El hecho de que haya analfabetos o personas sin teléfono no nos lleva a condenar la escritura o las telecomunicaciones, por el contrario, somos estimulados a desenvolver la educación primaria y a extender las redes telefónicas. Debería ocurrir lo mismo con el ciberespacio. (p. 237) (subrayado nuestro, traducción propia)

Por otro lado, en Europa, a finales de marzo de 2014 el Parlamento de la Unión Europea votó a favor de la neutralidad de red la propuesta en curso de regulación única de los servicios de comunicación electrónica, en lo que denominan “European single market for electronic communications". En una de las enmiendas votadas, refiriéndose a este principio, el Parlamento afirmó lo siguiente:

The existing regulatory framework aims at promoting the ability of end-users to access and distribute information or run applications and services of their choice. Recently, however, the report of the Body of European Regulators for Electronic Communications (BEREC) on traffic management practices published in May 2012 and study, commissioned by the Executive Agency for Consumers and Health and published in December 2012, on the functioning of the market of internet access and provision from a consumer perspective, showed that a 
significant number of end-users are affected by traffic management practices which block or slow down specific applications. These tendencies require clear rules at the Union level to maintain the open internet and to avoid fragmentation of the single market resulting from individual Member States' measures.

Sin embargo, la ley debe pasar aún por el Consejo de Ministros para poder entrar en vigor.

Ya en Brasil, la Agencia Nacional de Telecomunicaciones, Anatel, se posicionó en pro del principio de la net neutrality, o neutralidad de red, conforme es Ilamado en Brasil, al aprobar, mediante la Resolución 614 de mayo de 2013, la Regulación de Servicio de Comunicación Multimedia, resaltando en su artículo 75 , al igual que de manera amplia, que "las Prestadoras de Servicio de Comunicación Multimedia deben respetar la neutralidad de red, conforme reglamentación, en los términos de la legislación". Aun así, aparentemente Anatel se contuvo de entrar en pormenores pues está aguardando la promulgación del actual proyecto de ley ya mencionado arriba, esto es, el Marco Civil de Internet. Tal proyecto, en su artículo 3, inciso IV, dispone que la disciplina del uso de Internet en Brasil tiene como uno de sus fundamentos la "preservación y garantía de la neutralidad de red"; e inclusive, en su artículo 24, inciso VII, se constituye como una de las directrices para la actuación de la Unión, de los Estados, del Distrito Federal y de los Municipios en el desarrollo de Internet en Brasil la "optimización de la infraestructura de las redes y el estímulo a la implantación de centros de almacenamiento, gerenciamiento y diseminación de datos en el País, promoviendo la calidad técnica, la innovación y la difusión de las aplicaciones de Internet, sin perjuicio de la abertura, la neutralidad y la naturaleza participativa”. En Brasil, así, si es aprobado dicho proyecto en estos términos, o que sea de otra forma pero garantizando la neutralidad de red, se caminará al mismo nivel de las legislaciones holandesa y chilena, aprobadas por el Parlamento holandés y por la Cámara chilena, respectivamente, en 2011 y $2010 .^{5}$ El texto de la Ley chilena 18168, con la nueva redacción dada por la Ley 20643 de 2010, por ejemplo, proclama en el artículo $24, \mathrm{~h}, \mathrm{a})$, que:

No podrán arbitrariamente bloquear, interferir, discriminar, entorpecer ni restringir el derecho de cualquier usuario de Internet para utilizar, enviar, recibir u ofrecer cualquier contenido, aplicación el servicio legal a través de Internet, así como cualquier otro tipo de actividad o uso legal realizado a través de la red. En este sentido, deberán ofrecer a cada usuario un servicio de acceso a Internet o de conectividad al proveedor de acceso a Internet, según corresponda, que no distinga arbitrariamente contenidos, aplicaciones o servicios, basados en la fuente de origen o propiedad de estos, habida cuenta de las distintas configuraciones de la conexión a Internet según el contrato vigente con los usuarios. Traducción propia

La referida ley, en este sentido, promueve la neutralidad de red al rechazar cualquier acto de la empresa proveedora de servicio que impida al usuario tener acceso integral al contenido de Internet. Así, la importancia de tal principio se

Consultar http://www.theregister.co.uk/2011/06/09/netherlands_1st_ european_country_to_adopt_net_neutrality/ y http://www.camara.cl/ prensa/noticias_detalle.aspx?prmid=38191 
da en la medida en que delinea un ambiente de libre acceso a la información, al conocimiento y a los servicios y productos ofrecidos online. La distinción arbitraria de contenidos, informaciones y servicios no traería ningún beneficio al usuario, solamente a las empresas prestadoras de cara a la posibilidad de estas de negociar la distribución del contenido online con los consumidores y con las demás empresas.

Acompañamos, así, el entendimiento de Tim Berners-Lee (2010, p. 84), creador de World Wide Web, quien afirma, en el artículo publicado en la revista americana Scientific American, en defensa del principio de la neutralidad de red, que "a neutral communications medium is the basis of a fair, competitive market economy, of democracy, and of science".

\section{LA ECONOMÍA Y LA BASE INMATERIAL DE LA SOCIEDAD}

Caminar en este sentido, a nuestro parecer, es direccionarse en pro de los beneficios traídos por las tecnologías de la información y comunicación sin el perjuicio de promover los desajustes que puedan resultar de una lógica capitalista que desequilibra las capacidades, de individuo a individuo, o de sociedad a sociedad, de condiciones de fruición de una verdadera comunidad global del conocimiento, cuyas ventajas socioeconómicas, políticas y culturales estimulan la difusión de información irrestricta en el globo. Así, existe la posibilidad de experimentar con plenitud las proposiciones expuestas por Lévy (2001), desde el punto de vista económico, como un "extraordinario fenómeno de transformación social”, a saber:

1) La economía se torna una libre economía de la información y de los conocimientos.

2) Consecuentemente, la inteligencia colectiva, esto es, el proceso social de cambio y de producción de los conocimientos, se torna una especie de economía de mercado ampliada. Simétricamente, esa nueva economía de mercado se fundamenta progresivamente en la inteligencia colectiva.

3) Es en el ciberespacio que se entrelaza, ya hoy, la convergencia progresiva de un mercado que se libera y virtualiza, de un lado, y el proceso de inteligencia colectiva y de crecimiento dinámico del saber que caracteriza a la especie humana, de otro.

4) El punto de unión entre la economía y la inteligencia, el centro secreto de la sociedad humana del futuro es probablemente la capacidad de escucha y de manipulación de la conciencia colectiva que fluctúa en los millones de canales del ciberespacio [...]. Las instituciones, los Estados, los partidos, las administraciones públicas, las universidades, los museos, las empresas, las asociaciones, los grupos de interés, los individuos, todos aquellos que descuiden los estudios de las mejores maneras de insertarse en los procesos de inteligencia colectiva y de distribución de la atención que están en juego en un ciberespacio planetario no podrán más pretender desempeñar un papel importante en el mundo que vendrá. (pp. 59-60). Traducción propia

Para este autor, el concepto de riqueza es el "espacio de conciencia convenientemente ex- 
plotado" (Lévy, 2001, p. 60), comprendiendo tres aspectos o polos: la invención, la explotación o traducción económica de la invención, y el medio favorable a la invención y su explotación económica (Lévy, 2001, p. 61). Entramos, así, a nuestro parecer, en una lógica de la abundancia permanente, teniendo presente que las invenciones, las ideas, advenidas del espíritu colectivo, son infinitas. La lógica de la escasez, por tanto, acompañando al autor, solo puede ser manejada mientras se inserte en cierta dimensión espaciotemporal. A largo plazo, todas las bases materiales pueden ser modificadas, gracias a la concepción inmaterial e infinita del conocimiento y de las ideas e invenciones. Así, Lévy llega a afirmar que la sociedad no tiene una base material puesto que esta es muy reivindicada por la economía, pero sí una base espiritual, "pues todo viene del espíritu colectivo de la humanidad y se materializa a lo largo de un proceso social de expansión y de complejización progresiva" (Lévy, 2001, p. 63), fundado en la infinidad de posibilidades inherentes a la producción de ideas.

La base inmaterial de la sociedad, de esta forma, se convierte en un factor esencial para la producción y reproducción del orden económico vigente. Fortalecida por las tecnologías de información y comunicación, resulta de un proceso de interacción social realizado de forma y velocidad jamás vistos.

El derecho, por tanto, no puede escapar de este camino universalizante y cada vez más edificador del espíritu colectivo, bajo el riesgo de desacompañar el desarrollo económico, social, político y cultural promovido por la actual sociedad del conocimiento y sus instrumentos de interacción y donación de sentido, evidentemente globalizados. Como ya resaltamos en otro momento, "el derecho no necesita estar estratificado para que exista, pero sí debe estar soportado en el llamado espíritu objetivo para que sea mínimamente concreto en su aplicación" (Matsushita, 2012, p. 31).

La defensa en pro del principio de neutralidad de red es también en pro de la oportunidad de emancipación, individual, colectiva y considerada igualmente entre todos los del globo, con la finalidad de dignificar al hombre y todos los hombres, en un espíritu de fraternidad. Esto porque la dimensión fraterna de los derechos humanos conduce necesariamente a la dimensión propia de la globalización y de la democratización del acceso a la información, ya que habla sobre la garantía para todos de la construcción de un proceso de autonomía e independencia, tanto material como inmaterial. Tal garantía será firmada solamente mediante el compromiso de una abstención mediante el establecimiento de los límites del mercado a la adquisición de información y la producción de conocimiento, caminando, así, en la esfera del jushumanismo normativo:

Es en ese espíritu del humanismo, antropofilíaco, donde el hombre es desplazado del centro de las cosas, para ser parte del medio difuso del todo, que puede patrimonializar los derechos humanos en las múltiples dimensiones del hombre, de la colectividad y de la humanidad, incorporando el respectivo patrimonio subjetivo y tornándose de derecho y de hecho propiedad apta para el pleno ejercicio y concre- 
tización, incluso forzada, por medio de la tutela jurisdiccional. (Matsushita, 2012, p. 161). Traducción propia

La libre circulación de ideas, consecuentemente la libre circulación económica, hace parte del patrimonio subjetivo según la teoría mencionada, pues entendemos que los resultados advenedizos del capitalismo con base en este presupuesto y en la respectiva función social, tienden a traer incontables beneficios a la sociedad, inclusive, a todos, el deseado derecho objetivo de la dignidad de la persona humana. Por tanto, vemos como un contrasentido la estipulación de límites de contenido por las empresas ofertantes del servicio de Internet, según el juicio norteamericano aquí expuesto, ya que el propio capitalismo aprecia el ejercicio libre y sin riendas de la economía, lo que será reducido si las oportunidades de generación, producción y distribución de conocimiento fueran limitadas. La ventaja a corto plazo para tales empresas de obtención inmediata de mayor lucro debe ser excluida por el Estado, sirviendo este como garante del avance en la universalización del conocimiento, en pro del sistema capitalista y, al mismo tiempo, en pro de la dignidad humana.

En otras palabras, se debe promover y dejar promover el desarrollo del capitalismo y de sus tecnologías advenedizas, en el sentido de avanzar hacia la perspectiva de los derechos humanos fundamentales, evitando, de esta forma, un retroceso de cara a los beneficios y garantías conquistados, proyectando el sistema económico siempre para el bien humanista, evitándose la lógica del lucro irresponsable. Así, vale la pena resaltar que defendemos el ejercicio del capitalismo de forma libre, no obstante desde que sea conforme con la dignidad de la persona humana y los derechos incurridos.

\section{CONCLUSIONES}

Las tecnologías de la información forman un inmenso cabal de oportunidades para el desarroIlo de la sociedad en todos los aspectos sociales, culturales, políticos y económicos, capaz de auxiliar en la promoción de los derechos humanos y fundamentales en pro de la dignidad de la persona humana. Decimos esto, pues la oportunidad de emancipación individual y colectiva es inmediata gracias a la difusión constante y permanente de información y conocimiento, permitida y producida a través de las plataformas de comunicación, mayoritariamente de Internet.

El mercado, en la sociedad de la información, se condiciona a este nuevo ambiente haciendo flexible el valor agregado de las empresas y de sus productos para el ambiente virtual, de cambio de conocimiento y de información, promoviendo, de esta forma, una nueva estructura económica. Esa estructura reconoce no solamente la producción de bienes materiales, también lo inmaterial, identificado con el propio contenido que circula en los nuevos medios de comunicación, muchas veces creado y reciclado por los propios consumidores. Surge la coyuntura de mercados paralelos a aquellos de los grandes conglomerados comerciales, con servicios y productos ampliamente comercializados a bajos costos, democratizando el acceso. 
La economía pasa a no basarse más en la lógica de la escasez de recursos materiales, pues la información y el conocimiento son producidos en abundancia, ocasionando la expansión -a una velocidad sin precedentes- de una nueva economía digital.

En este sentido, para el mantenimiento de los factores benéficos advenedizos con el avance de las tecnologías de información y comunicación, se hace urgente la preservación del principio de la neutralidad de red, para garantizar el acceso amplio y democrático a todo lo que se encuentra producido en la red, en pro de una verdadera globalización, sin estar condicionada a las divisiones políticas, económicas y sociales a que estamos sometidos hace siglos.

Se concluye así que, en esta nueva economía, en la que el poder político y económico se encuentran cada vez más difusos, es necesario que prestemos atención al mantenimiento del respeto y observancia de los derechos humanos fundamentales, donde se encuentra inclusive el derecho a la información. Por tanto, tomando en cuenta el pensamiento jushumanista normativo de garantizar para todos en el planeta el derecho a la dignidad de la persona humana, vemos las tecnologías referidas como esenciales para la conducción de una sociedad cada vez más libre, justa y solidaria, partiendo todos del espíritu objetivo del humanismo como principio rector de la libertad de invención y de participación global de ideas y prácticas democráticas, contrarias a la dictadura del capitalismo sin riendas.

\section{Referencias}

Anderson, C. (2012). Makers. The new industrial revolution. United States of America: Crown Business.

Bauman, Z. (1999). Modernidade e ambivalência. Rio de Janeiro: Jorge Zahar Editor.

Berners-Lee, T. (2010). Long Live the Web: A Call for Continued Open Standards and Neutrality. Scientific American, 303 (6).

Bonavides, P. (2004). Curso de Direito Constitucional (15. ${ }^{\mathrm{a}}$ ed). São Paulo: Editora Malheiros.

Comparato, F. K. (2008). A afirmação histórica dos direitos humanos (6. ${ }^{a}$ ed.). São Paulo: Saraiva.

Held, D. y McGrew, A. (2001). Prós e contras da globalização. Rio de Janeiro: Jorge Zahar.

Lévy, P. (2001). A conexão planetária: o mercado, o ciberespaço, a consciência. São Paulo: Editora 34.

- (1999). Cibercultura. São Paulo: Editora 34.

Matsushita, T. (2012). O jus-humanismo normativo - expressão do princípio absoluto da proporcionalidade. 206 f. Tese (Doutorado em Direito) - Pontifícia Universidade Católica de São Paulo, 180-190. 
Cibergrafía

Debate sobre Marco Civil de Internet con Marilena Chauíy Sérgio Amadeu da Silveira (2013). Programa TV Fórum. Recuperado de http:// www.youtube.com/watch?v=uQwYIhwvwWQ

Estes, A. C. (2014). The Grim Future of a World Without Net Neutrality. Gismodo. Recuperado de http://gizmodo.com/the-grim-future-of-a-world-without-net-neutrality-1501161513

Guerra Filho, W. S. (2013). Para pensar a eclosão do movimento das multidões. Jornal Estado de Direito. Recuperado de http://www. estadodedireito.com.br/2013/08/06/para-pensar-a-eclosao-do-movimento-das-multidoes/
Pepper, R. (2007). Network Neutrality: Avoiding a Net Loss. TechNewsWorld. Recuperado de http://www.technewsworld.com/ story/56272.html

Villaverde, J. (2014). Dilma anunciará “Marco Civil mundial' em evento na capital paulista. Redação Link. Recuperado de http://blogs. estadao.com.br/link/dilma-anunciara-marco-civil-mundial-em-evento-na-capital-paulista/

Zajac, A. y Shields, T. (2014). Verizon Wins Net Neutrality Court Ruling Against FCC. Bloomberg. Recuperado de http://www.bloomberg. com/news/2014-01-14/verizon-wins-net-neutrality-court-ruling-against-fcc.html 